\title{
Being a beast machine: The origins of selfhood in control-oriented interoceptive inference
}

\author{
Anil K Seth \\ Sackler Centre for Consciousness Science \\ School of Engineering and Informatics \\ University of Sussex \\ Brighton, BN1 9QJ \\ United Kingdom \\ a.k.seth@sussex.ac.uk \\ www.anilseth.com \\ @anilkseth
}

20th Sept 2017

\section{Summary}

Throughout his career Andy Clark has shaped how scientists and philosophers think about the role of representation in action, perception, and cognition. In the latest iteration of this debate he has foregrounded the influential perspective of 'predictive processing', which sees perception as a process of action-oriented 'best guessing' (inference) about the causes of noisy and ambiguous sensory signals, and which involves the brain inducing 'generative' models of how hidden causes mediate the effects of actions on sensory signals. Here, I will develop this position in the context of interoception (the sense of the body from within) and physiological regulation. A key idea here, which recalls 20th century cybernetic theory, is that interoceptive inference is targeted towards maintaining physiological homeostasis rather than inducing complete and accurate internal models of an external state-of-affairs. I explore how this perspective helps connect control-oriented interoceptive inference to phenomenological properties of embodied selfhood and subjectivity. The upshot echoes (or perhaps subverts) a classic philosophical trope of the Enlightenment philosopher Julien La Mettrie: to find the origins of our conscious selves in our nature as beast machines.

Keywords: interoceptive inference, interoception, cybernetics, allostasis, selfhood, predictive processing

\subsection{In pursuit of a philosopher}

For more than twenty years my career has been defined by the unsuccessful geographical and intellectual pursuit of the philosopher Andy Clark. My early undergraduate days, studying experimental psychology at Cambridge in the 1990s, were enlivened on reading his seminal Microcognition (Clark, 1989). Notions of distributed and graded representations, dynamically encoded by neuronal populations, made an immediate impact in a syllabus otherwise full of animal learning theory and box-and-arrow models of cognitive function. Inspired, I made my way to the University of Sussex for a Master's degree, and subsequently a doctorate. I wanted to be part of the School of Cognitive and Computing Sciences, to breathe its interdisciplinary mix of psychology, philosophy, and artificial intelligence - and to be where (I thought) Andy Clark was leading the charge. 
He was indeed leading the charge, but no longer from Sussex. By the time I arrived in 1995 he had departed to direct the philosophy, neuroscience, and psychology programme at Washington University. So it was from a distance that I devoured his next salvo, Being There (Clark, 1997). By now, well embarked on my D.Phil. (Ph.D.) in evolutionary robotics and artificial life (Seth, 2000), I was eagerly soaking up the insights offered by embodied and enactive approaches to cognitive science. As Microcognition had done for neural networks, Being There crystallized the insights of these movements so elegantly that - at least for me - it was suddenly difficult to imagine otherwise. Of course brains are embodied and bodies are embedded, so that feats of cognition and behavior can only be explained by appeal to these continually unfolding interactions.

At this point I nearly caught up with the elusive Clark. He had returned to Sussex just in time to act as examiner for my thesis, around spring of 2001, but his feet barely touched the ground before he was off to Edinburgh to take up his current prestigious professorship. And now it was my turn to go to the USA, to the Neurosciences Institute in San Diego, to immerse myself in the more neuroscientific aspects of cognitive science and consciousness research.

On returning to Sussex several years later, I became increasingly interested in the framework of 'predictive processing' in its various guises, and especially in their relevance to the neuroscience of consciousness (Seth, 2009, 2014). Once again, Andy Clark stole the show, this time with his landmark discussion paper Whatever next? (Clark, 2013), followed more recently by Surfing Uncertainty (Clark, 2016) which together with Jakob Hohwy's The Predictive Mind (Hohwy, 2013) and Karl Friston's prodigious publications on the free energy principle [e.g., (Friston, 2009, 2010), see also (Buckley, McGregor, Kim, \& Seth, (in press))] have set the agenda for a good swathe of $21^{\text {st }}$ century cognitive neuroscience.

Much of Clark's oeuvre, including the works mentioned above, can be understood in terms of examining the nature and role of mental representations in cognition and behavior. His contributions have continually advanced the discussion between those who emphasize internal structures and processes, and those who see the constitutive basis of cognition as extruded into the world. I often -but not always- find myself in agreement with the middle ground that he adroitly articulates. In this middle ground, it remains sensible to talk in terms of neurally encoded representations, but only within the larger context of brain-body-world interactions that support and structure adaptive behavior.

My aim in this chapter is therefore not to critique Clark, at least not directly. Instead I will outline a set of ideas which integrates across some of the same themes that he has developed over the last 20 years, primarily from Being There and Surfing Uncertainty. My focus will not be on the nature of mental representation, but instead on the relevance of these ideas for understanding the neurocognitive underpinnings of subjective experiences of embodiment and selfhood. The upshot is a deeply physiological account of embodied experience, echoing influential lines of thinking in cybernetics (Conant \& Ashby, 1970) and autopoeisis (Maturana \& Varela, 1980), in which basic experiences of selfhood are grounded in predictive mechanisms geared towards ensuring the survival of the organism. The view that emerges speaks to long-standing debates about the relationship between life and mind (Godfrey-Smith, 1996) and re-animates an old argument about our nature as 'beast machines' or 'man machines' (Rosenfield, 1968; Shugg, 1968). In setting this focus, I hope to push the discussion a little further towards consciousness as an explanatory target - something that over his career Andy Clark has orbited but rarely attempted to assault directly. Instead of merely 'creeping up on consciousness' (Surfing Uncertainty, Chapter 7) perhaps now is the time for Andy to fully apply his characteristic lucidity to this most thorny of challenges. To me, at least, he already has the tools he needs. 
"We are not cognitive couch potatoes idly awaiting the next 'input', so much as proactive predictavores - nature's own guessing machines forever trying to stay one step ahead by surfing the incoming waves of sensory stimulation" (Surfing Uncertainty, p.52)

I will first summarize - very briefly - the broad outlines of predictive processing, on which the subsequent excursions into interoception and the phenomenology of selfhood rely. (Much of this material is also covered, and with greater authority, in Karl Friston's chapter in this volume - a chapter I will make occasional reference to where useful ${ }^{1}$.) The basic idea behind predictive processing is simple and follows von Helmholtz's early conceptions of perception as 'unconscious inference' (von Helmholtz, 1867). It is that perception is in the business of inferring the causes of the signals that continually impinge on our sensory surfaces: our eyes and our ears, but also our other senses including those originating from within the body (interoception). 'Inference' here simply means that perception involves a probabilistic process of 'best guessing', in which incoming data is interpreted in the light of (typically unconscious) prior expectations or beliefs. This kind of reasoning - or inference - is necessary since there are complex, nonlinear, noisy and ambiguous mappings between sensory signals and their (hidden) causes, whether they originate from the world or from the body. More formally, this kind of inference implies a Bayesian process in which prior beliefs and prior beliefs are combined with sensory data (the likelihood) to furnish a 'best guess' - or Bayesian posterior - specifying the most likely causes of current sensory inputs (Clark, 2013).

Operationally, the simplest implementation of predictive processing (PP) for perception is via 'predictive coding'. In predictive coding schemes, neuronal representations in higher or deeper levels of neuronal hierarchies generate predictions about representations in lower levels. These descending predictions are compared with lower-level representations to form prediction errors, which are passed back up the hierarchy to update higher-level representations (Rao \& Ballard, 1999). The recurrent exchange of signals between adjacent levels resolves prediction error at each and every level, resulting in a hierarchically deep neurally-encoded 'explanation' for sensory inputs. In computational terms, the activity of neuronal populations is assumed to encode Bayesian beliefs or probability distributions over states in the world that cause sensations (Seth \& Friston, 2016). An important implication of this view is that perception involves the deployment of generative models which map from (hidden) causes to sensory signals. More informally, to perceive something implies the ability to imagine it.

One critical aspect of this process, for present purposes, is that PP does not entail or assume that the function of perception is to recover a veridical and action-independent representation of an external (or internal) world. Instead, predictive perception enables an organism to engage with its environment (which includes its own body) in ways which best support its goals, including - most fundamentally - continuing physiological integrity and survival. Clark calls this action-oriented predictive processing (Clark, 2013) and there are of course deeper roots tracing back to Gibsonian ecological psychology (Gibson, 1979) and beyond. Appreciating this point requires noting two additional aspects of PP: precision weighting and active inference. (Again, the chapters by Karl Friston and Jakob Hohwy provide an excellent introduction to these concepts.)

\footnotetext{
${ }^{1}$ For those who have already read Friston's chapter, my approach to PP reflects a 'middle road' which starts from the idea of unconscious inference (like the 'low road') but moves quickly to the idea that perceptual inference of all kinds is mandated by a fundamental biological imperative to stay alive - which approaches Friston's 'high road'. See Friston (this volume).
} 
The influence of sensory signals on perceptual inference depends on the reliability or confidence afforded to these signals. Salient sensory signals, or signals expected to have high reliability, will have a greater influence on updating perceptual predictions. Within PP, the process of adjusting expectations about reliability or salience is called precision weighting. Sensory prediction errors are always precision-weighted, so that sensory data with higher precision-weighting will have greater influence. Statistically, given the standard assumption of Gaussian or normal distributions (bell curves), precision is simply 'inverse variance' - a signal with low (expected) precision is encoded by a relatively broad bell curve. It's worth emphasising that precision itself has to be inferred. That is, the brain has to optimize its estimates of the precision of sensory data, a process which can intuitively associated with attention (Feldman \& Friston, 2010).

Active inference emphasizes that prediction errors can be minimized either by updating predictions (as in standard predictive coding schemes), or by changing sensory inputs via action, to match predictions. For example, simple bodily actions like raising an arm can be understood as the minimization of proprioceptive prediction errors through engaging motor reflexes. The balance between these two processes depends on precision weighting. If sensory data are attributed with relatively low (expected) precision, they will have little influence on prior beliefs, so that these priors will instead be fulfilled through actions. This accounts neatly for sensory attenuation (i.e., 'inattention') during action execution (Brown, Adams, Parees, Edwards, \& Friston, 2013; Friston, Daunizeau, Kilner, \& Kiebel, 2010). It's worth noting here that active inference brings into view a close coupling between perception and action, moving significantly beyond early predictive coding models of perception as a purely passive process of best-guessing.

Precision weighting and active inference mean that perception will always be shaped by functional goals: perceiving the world (and the self) not 'as it is', but as it is useful to do so. This in turn means that predictive models can be utilized for control, as perceptual predictions become fulfilled rather than updated. To make this clear, think of catching a cricket ball. One might think the best way to do this is to figure out where the ball is going to land and then run there as fast as possible. But this is not, in fact, a good strategy, and is not what expert fielders do. Instead, the best strategy is to keep moving so that the ball always 'looks the same' in a particular way (specifically, so that the angle of gaze elevation to the ball increases, but at a monotonically decreasing rate). This guarantees interception with the ball, and is in fact what accomplished fielders seem to do (McLeod, Reed, \& Dienes, 2003). This is an excellent example of perceptual control, in which the machinery of perception is leveraged to control (the hidden causes of) a perceptual variable, in other words to regulate something, and not to discover some action-independent state-of-affairs. Indeed, the often overlooked 'perceptual control theory' argues that control systems control what they sense, not what they do (W. T. Powers, 1973). It's worth noting that accomplished fielders are typically not aware of the perceptual control strategy that they are following, often believing that they are doing something entirely different. Inexpert fielders may of course both believe they are doing something different, and also be doing something different again, when they fail to catch the ball. This underlines the unreliability of first-person intuitions about perception and its relation to action.

Just as nonlinear and noisy relationships between hidden causes and sensory data require generative models and inference for perception, perceptual control in nontrivial environments also requires a model to predict the sensory consequence of actions. This recalls the influential (and until recently also overlooked) tradition of cybernetics in artificial intelligence and engineering (Ashby, 1956). A key paper in this canon argues, and attempts to show mathematically, that "every good regulator of a system must be a model of that system" (Conant \& Ashby, 1970). Intuitively this makes sense especially when circumstances require anticipatory control: in order to prevent a controlled variable departing from its desired range, it is helpful to predict its trajectory under upcoming or anticipated perturbations and deploy appropriate 
responses in advance. For instance: the best way to avoid getting wet is to not go out into the rain. For present purposes, a more appropriate example is found in the relationship between interoception and physiological regulation. The desirable state of being alive imposes tight bounds on many physiological quantities (heart rate, blood osmolality, and so on - what in the cybernetic literature are called 'essential variables' (Ashby, 1952)), such that it may be incompatible with survival to wait until these quantities go out of bounds before deploying compensatory responses. In these situations, anticipatory control is needed: allostasis (the process of achieving homeostasis) rather than homeostasis per se (Sterling, 2012).

Summarizing so far, starting from the Helmholtzian view of perception as (unconscious) inference, we've encountered the idea that perception involves a balance between what can now be called epistemic active inference, in which actions are deployed to refine generative models of the hidden causes of sensory signals (i.e., to come up with better predictive models of the world and the self), and instrumental or control-oriented active inference, in which actions are deployed in order to regulate the hidden causes of sensory signals - to keep them 'within bounds'. The use of action to either refine a model, or deploy it for regulation, maps onto the classic explore/exploit trade-off in reinforcement learning theory (Dayan \& Daw, 2008) (see also Friston, this volume). While good regulation may generally require a good model (especially when the mappings between hidden causes and sensory signals are noisy and nonlinear), at any particular time, and depending on the current physiological importance of regulating a particular hidden cause (e.g., contrast catching a cricket ball versus keeping heartbeat variability within bounds), the balance between refining the model and using the model for control will change according to context. Moreover, what constitutes a 'good' model is now not necessarily one that recapitulates the world (or body) as it is, but one that infers hidden causes (and their behavior under different actions) as it is useful for the organism to do so, given the biological imperative of staying alive.

\subsection{Interoceptive inference}

"Our ongoing sense of embodiment ... depends upon accommodating the full (interoceptive and exteroceptive) sensory barrage using a generative model whose dimensions crucially track aspects of ourselves" (Surfing Uncertainty, p. 231).

Perceptual inference has mainly been studied in the context of exteroceptive sensation - those signals which impinge on the outward-facing sensory surfaces of the body, relating to hidden causes in the external world. Vision in particular remains a focus, not least because of the relative ease with which visual stimuli can be constructed and presented, and also thanks to the encouragingly hierarchical appearance of the relevant functional neuroanatomy (Felleman \& Van Essen, 1991; Markov et al., 2013). But if predictive processing is to fulfil its promise as a general theory of brain function - and perhaps conscious experience the perceptual modalities of proprioception and interoception must also be accommodated in ways which shed light on their function and phenomenology.

Proprioception, which reports body posture and motion, provides an important example of active inference. As already mentioned, action elicitation can be thought of as fulfilment of proprioceptive predictions (Friston et al., 2010), providing an alternative to optimality-based approaches to motor control based on coupled forward and inverse models (Wolpert \& Kawato, 1998). As we will see below, interoception too can be brought within a PP framework, delivering a number of insights into experiences of emotion, embodiment, and selfhood, and their relation to allostatic physiological regulation (Seth, 2013, 2015a; Seth \& Friston, 2016; Seth, Suzuki, \& Critchley, 2011); see also (Barrett \& Simmons, 2015; Pezzulo, Rigoli, \& Friston, 2015; Stephan et al., 2016). 
Interoception typically refers to sensations originating from internal bodily organs, transmitted to the central nervous system, that reflect the functional integrity of those organs - as well as, potentially, the functioning of the body as whole (Craig, 2002; Critchley \& Harrison, 2013). Interoceptive signaling has long been associated with emotional experience, with early conceptions reaching back to the James-Lange theory of emotion as the perception of change in physiological condition, rather than as a direct response to an external stimulus (James, 1894). Later versions of this idea recognized the importance of context, giving rise to influential 'two-factor' theories in which the occurrence of an emotion depends on the cognitive interpretation of perceptions of physiological change (Schachter \& Singer, 1962) and not simply on the detection of any specific physiological signature (where 'cognitive' here simply refers to higher-level functions which, at least at the time, were taken to operate largely independently of 'low level' mechanisms of perception).

Only relatively recently has the conceptual machinery of PP been brought to bear on this issue, dissolving the implicit boundary between perception and cognition implied by two-factor theories, and - by straightforward analogy with PP accounts of vision and of other exteroceptive modalities - conceptualizing emotional feelings as the joint content of predictions about the (hidden) causes of interoceptive signals across multiple hierarchical levels (Barrett \& Simmons, 2015; Seth, 2013; Seth et al., 2011). Importantly, this means that emotional content can be influenced by predictions emanating not only from specialized interoceptive brain regions (e.g., the insular cortex), but also from multimodal or amodal predictions that have interoceptive or autonomic consequences. As Clark puts it:

“... interoceptive, proprioceptive, and exteroceptive predictions are constructed differently in different contexts, and each provides guidance to the other. A single inferential process here integrates all these sources of information, generating a context-reflecting amalgam that is experienced as emotion" (Surfing Uncertainty, p.234).

This perspective, which can be called the interoceptive inference theory of emotion (Seth, 2013, 2015a), marks a shift from 'classical' theories of emotion in which each emotion is caused by its own dedicated neural circuit (Tracy \& Randles, 2011) towards a view in which emotions are constructed by neural processes, such as perceptual inference and memory, which reflect principles of structural and functional organization that generalize beyond emotion itself (Barrett \& Satpute, 2017).

There is an important twist to the bringing together of PP, interoception, and emotion, which loops back to the distinction between epistemic and instrumental (control-oriented) active inference, introduced in the previous section in the context of cybernetics and the predictive regulation of essential variables. As compared to exteroceptive modalities like vision, interoceptive inference is likely to place greater emphasis on control than on discovery, given the importance of effective regulation of physiological variables. In fact, one can think of the entirety of the brain's predictive apparatus as being geared, ultimately, towards effective physiological regulation - towards allostatic control (Barrett \& Simmons, 2015; Pezzulo et al., 2015; Seth, 2015a; Seth \& Friston, 2016) ${ }^{2}$. In this view, even (apparently epistemic) visual or auditory inference will be inflected by the allostatic relevance of the inferred hidden causes - you may be more likely to interpret a squeaking window as being caused by a thief (rather than by the wind) if your

\footnotetext{
2 Here is where we start cutting across to Karl Friston's high road - inasmuch as allostatic control essential for existing (which may not be true for all things that exist). See Friston (this volume).
} 
interoceptive context (and hence the relevance of any sensory signal for allostasis) has been altered by having just watched a horror movie (Pezzulo, 2014).

Even more generally, the fundamental importance of physiological viability suggests that the brain's predictive machinery has phylogenetic and ontogenetic roots in allostasis. That is, predictive perception evolved (and develops) for the purposes of allostatic regulation. This idea brings us close to the territory of the 'free energy principle' (FEP) (Friston, 2010) which is undoubtedly the most ambitious of all theoretical frameworks related to PP. According to the FEP, all organisms, simply by virtue of their existence, are mandated to minimize the entropy, dispersion, or 'atypicality' of their constituent (or sensory) states ${ }^{3}$. In other words, organisms inhabit states in which they expect to be in. This basic condition on the nature of living organisms stems from their need to resist the tendency to disorder implied by the second law of thermodynamics, and is taken to apply to all features of living systems - i.e., to gross morphology as well as to the fine details of cortical microcircuits - as well as at all timescales, from the phylogenetic to the neuronal. As Friston and Stephan put it, "all the quantities that can change; i.e., that are part of the system, will change to minimize free-energy" (Friston \& Stephan, 2007) (p.427).

Free energy appears here as a mathematical upper bound on the entropy of sensory states, which (unlike sensory entropy per se) is a quantity that can in principle be computed or measured by an organism. In other words, unlike the entropy of sensory states, free energy is a quantity that can be computed and hence minimized (see Friston, this volume). Indeed, under the useful assumption that sensory variables can be approximated by Gaussian distributions, free energy becomes equivalent to prediction error as described above. While the mathematical details are complex [encompassing elements from statistical mechanics, thermodynamics, and machine learning; see (Buckley et al., (in press)) for a recent review], the basic message is simple. It is that the computational machinery of predictive perception - and more importantly predictive regulation through active inference - can be understood to stem from basic physical principles that apply to living systems (perhaps, even, to all systems that can be said to exist), which entail that such systems must maintain themselves within a restricted repertoire of states. Intriguingly, this view suggests deep and perhaps obligatory connections between life and mind - an idea we will return to at the close of this chapter (Godfrey-Smith, 1996; Thompson, 2010).

\subsection{Conscious self: the experience of being you}

"This multilayered texture, in which a world of external causes and opportunities for organismsalient action is presented to a creature in a way constantly intermingled with a grip upon its own bodily condition may lie at the heart of that ever-elusive, and ever-familiar, beast that we call 'conscious experience' "' (Surfing Uncertainty, p.239)

How can PP explain the variety of perceptual experiences that we enjoy (or endure)? In particular, can PP account for the qualitative differences between perceptual experiences of the external world, and selfrelated experiences such as emotion and experiences of having, and being, a body? It is not enough to say that emotional experiences are the way they are (and are different to e.g., visual experiences) because they involve predictions about interoceptive signals rather than predictions about visual signals. Such an

\footnotetext{
${ }^{3}$ For present purposes we can treat sensory states and constituent states of an organism as equivalent. See (Buckley et al., (in press)) for the mathematical details. At this point in the story we are rather close to Friston's 'high road' (see Friston, this volume).
} 
'explanation' is no more informative than saying visual experience is the way it is because it activates occipital rather than insular cortex. A more useful approach is to consider the nature of the predictions (i.e., epistemic versus instrumental) associated with each modality.

Consider one prominent aspect of visual phenomenology: objecthood. Looking past my desk through the window out to the campus beyond, my visual experience seems comprised, to a large extent, of objects (computer screen, window frame, tree) and the spaces between them. What defines the phenomenology of objecthood? One simple feature is that the experience of an object seems to include the existence of surfaces that are not directly represented in sensory data. When we perceive an object, we perceive it as having an external existence - with a 'back and sides' - and not simply as a 'perspectival take' on an external scene (Noe, 2004).

The phenomenology of emotion and of embodiment is not like this. Emotions are not experienced as existing in the same way that (visual) objects are: for instance, emotions do not occupy a volume in space, nor do they have a back or sides. Experiences of embodiment are perhaps even more challenging to describe. Through some modalities (again, notably vision) the body can be perceived as a specific object. I may perceive the hand in front of me as my hand - an experience of body ownership which can extend to the entire body-as-object (Blanke, Slater, \& Serino, 2015; Tsakiris, 2010). However, there is also an inchoate phenomenology of 'being a body' - a background experience of selfhood which shades into mood and emotion at one end and into experiences of body ownership at the other. This aspect of embodied experience does not involve a strong component of objecthood, but rather seems to reflect an evaluation of the physiological state of the organism and its future prospects given the current context.

Here's where things become rather speculative. I believe that the machinery of PP, and in particular of control-oriented interoceptive inference, can shed useful light on these aspects of phenomenology. A first clue is that experiences of the body as an object can be shaped not only by exteroceptive and proprioceptive signals, but also by interoceptive signals. For example, there is a version of the classic rubber-hand illusion (Botvinick \& Cohen, 1998) in which a virtual 'rubber hand' gently pulses either in-time or out-of-time with the heartbeat [the heart being a prominent source of interoceptive signaling, see (Critchley \& Harrison, 2013)]. Participants have a stronger sense of ownership of the virtual hand in the synchronous as compared to the asynchronous condition, indicating that perceptual inferences about body ownership are shaped by interoceptive as well as by exteroceptive and proprioceptive signals (Suzuki, Garfinkel, Critchley, \& Seth, 2013).

When it comes to the inchoate experiences of being a body, my conjecture is that its non-object-like phenomenology is linked to the control-oriented nature of interoceptive predictions. To explain why, let's consider which features of predictive perception could underpin a phenomenology of objecthood in general. In an influential body of work, Alva Noë and Kevin O'Regan associate objecthood - equivalent to what they call perceptual presence - with a 'mastery' of sensorimotor contingencies (O'Regan \& Noë, 2001). The idea is that we interpret sensory signals as being caused by a (really existing) object, when this process of interpretation involves knowledge (or mastery) of how sensory signals would respond given various actions. Intuitively, I perceive the mug in front of me as having a back, because my process of perception encompasses the knowledge that rotating the mug (or moving around it) would bring its back into view. In this way, their 'sensorimotor contingency theory' explains why objecthood associates with some perceptual phenomena (like the mug in front of me) but not with others (for example: clear blue skies and retinal afterimages). 
But what does 'knowledge' or 'mastery' of sensorimotor contingencies mean, in terms of neurocognitive mechanism? From the perspective of PP, knowledge about the sensory consequences of actions can be interpreted in terms of conditional or counterfactual predictions - i.e., predictions about how sensory signals would change were specific actions (e.g., eye movements) carried out (Friston, Adams, Perrinet, \& Breakspear, 2012). I previously suggested that perceptual presence - the phenomenology of objecthood arises when perceptual inference is based on a rich repertoire of counterfactual predictions, reflecting a 'mastery' of the relevant sensorimotor contingencies (Seth, 2014, 2015b). In other words, I become perceptually aware of the back of the mug (and thus the mug-as-a-whole benefits from the phenomenology of presence and objecthood) when the mechanisms of predictive perception, in my visual brain, are able to make a rich set of predictions about the sensory signals that I would encounter were I to make this-or-that action (picking it up, rotating it, and so on). When these conditions are satisfied, I consciously experience the mug as really existing in the world. ${ }^{4}$ This in turn requires that perceptual content is preferentially shaped by predictions rather than prediction errors, since counterfactual predictions are not actualized and so cannot generate prediction errors (at least, they cannot all be simultaneously actualized since that would require conflicting actions to be performed at the same time). An emerging body of evidence suggests that, indeed, perceptual content depends more on predictions than on prediction errors [see for example (Pinto, van Gaal, de Lange, Lamme, \& Seth, 2015; A. R. Powers, Mathys, \& Corlett, 2017)

Notice that in the example given above, the counterfactual predictions relevant to objecthood are to do with epistemic active inference. They are about actions that would, if executed, shed light on some external state-of-affairs: is it a real mug, or just a picture of a mug? For interoceptive predictions, the situation is different. Here, actions (either external or purely internal, like autonomic reflexes) are typically performed in order to control or regulate interoceptive sensations - and thereby to ensure allostatic integrity. Counterfactual predictions relevant to interoceptive inference would therefore be to do with predictions about the allostatic consequences of actions, not about discovering more about some particular state-ofaffairs. This returns us to the balance between refining a model (through epistemic inference) and deploying that model for the purposes of regulation (through instrumental inference).

It seems plausible that (instrumental) counterfactual predictions related to control would lead to a different phenomenology than (epistemic) counterfactual predictions related to discovery. Instead of underpinning a phenomenology of objecthood, control-oriented inference may generate a phenomenology related to the evaluation of the allostatic consequences of regulatory actions. It may not be too far-fetched to suggest that this kind of phenomenology would be close to the non-localised, non-object-based phenomenology associated with both mood and emotion, and with the basic self-related experience of being an embodied organism (Seth, 2015a; Seth \& Friston, 2016).

It may even be that certain disorders of self-experience can be understood in this way. For example, depersonalization disorder is characterized by a subjective sense of the 'unreality' of the self and world (Sierra \& David, 2011), a distressing condition which in its extreme form can manifest in the delusion belief

\footnotetext{
${ }^{4}$ Most expressions of sensorimotor contingency theory resist this kind of operationalisation, perhaps because it implies that perception depends on some sort of internal 'model', which is a position anathema to some philosophers and cognitive scientists wedded to the so-called enactive approach. See for example (Hutto \& Myin, 2013). This seems like a missed opportunity. PP, especially in its active inference formulation, offers both an understanding of perception and cognition that has ('enactive') embodied agent-interaction at its heart, and a process theory, or theories, which put flesh on these conceptual bones at computational and implementation levels of description. For further discussion see (Seth, 2014).
} 
that one is dead [Cotard syndrome, (Young \& Leafhead, 1996)]. One may speculate that this sense of subjective unreality may be a consequence of counterfactually-impoverished control-oriented interoceptive inference, in just the same way that visual percepts may lose their 'objecthood' when a rich set of counterfactual predictions is missing (Seth, 2014, 2015b). ${ }^{5}$

Future testing these ideas may call on the rapidly emerging field of 'computational psychiatry' in which computational models are fit to fine-grained data obtained from participants performing well-controlled behavioural tasks (Huys, Maia, \& Frank, 2016). Individual-specific parameters of these models (e.g., things like learning rates, or the precision allocated to new sensory data) can then be used to characterize symptom expression and behaviour in terms of altered predictive processing. These parameters can then also be used in neuroimaging experiments to identify relevant changes in brain activity, which can provide further insight into the mechanisms underlying psychiatric symptoms, as well as identifying potential targets for pharmacological intervention. In the current context, the key would be to operationalize the phenomenology of dissociation in ways which admit fine-grained (e.g., 'trial-by-trial') measurements, to which computational models of interoceptive inference could be applied. See (Stephan et al., 2016) for an exciting example in the context of fatigue and depression.

\subsection{Back to life, back to reality ${ }^{6}$}

'They're made out of meat'

'Meat?'

'Meat.'

Andy Clark begins Surfing Uncertainty with this snippet from Terry Bissom's Alien Nation (1991), to highlight the wonder of how 'mere meat' gives to "thinking, imaging, dreaming, and the whole smorgasbord of mentality, emotion, and intelligent action" (p. xiv). But the wonder is not so much that 'mere meat' can think, imagine, and dream. Rather, it is that the fundamental experience of being an embodied organism may turn out to be deeply rooted in the meat-based predictive machinery that evolved, develops, and operates from moment-to-moment in light of the fundamental biological imperative towards staying alive. Underneath the ramified levels of selfhood involving memories of the past and plans for the future, before the explicit sense of personal identity, beneath the ' $I$ ' and even prior to the emergence of a first-person perspective on the world and body, the origins of selfhood trace back to our nature as self-sustaining meat-machines that care (consciously and unconsciously) about their own persistence. And if all our perceptions and cognitions are inflected by this biological imperative to stay alive, then the whole smorgasbord of human experience and mental life arises because of, and not in spite of, our physiological reality.

These ideas weave together several distinct threads, both current and historical. Ideas about controloriented interoceptive inference draw on mid-twentieth century cybernetics (Ashby, 1952; Conant \& Ashby, 1970) and model-based control. The deep, perhaps obligatory, links to allostasis and physiological integrity find formal expression in the free energy principle (Friston, 2010) (see also Friston, this chapter)

\footnotetext{
5 I previously suggested that depersonalization disorder may arise when interoceptive predictions are abnormally imprecise (Seth et al., 2011). While this early idea remains to be tested, the present account may provide a more satisfying bridge between mechanism and phenomenology. These are topics for future research.

${ }^{6}$ A tribute, of course, to British R\&B 'funki dreds' Soul II Soul: https://www.youtube.com/watch?v=TB54dZkzZOY.
} 
and provide a compelling argument for a strong continuity between life and mind (Maturana \& Varela, 1980; Thompson, 2010). Ideas about the phenomenology of presence and objecthood draw on sensorimotor contingency theory (O'Regan \& Noë, 2001) when operationalised in terms of predictive perception (Seth, 2014, (in press)).

Most historically resonant are the roots to Enlightenment debates about the nature of the soul and the relevance of the body (Makari, 2016). For Descartes, non-human animals were 'beast machines' without souls or conscious experiences of any kind (at least without any kind of experience warranting moral status). Writing after Descartes, in 1748, Julien de La Mettrie took this Cartesian idea to its extreme: if animals are beast machines then so are humans, since humans are also animals [(de La Mettrie, 1748); see (Makari, 2016; Rosenfield, 1968)]. Therefore, the soul (if there is such a thing) is a (biological) machine - a conclusion which Descartes would have strongly resisted, as indeed did many people in La Mettrie's time. ${ }^{7}$ The story sketched in this chapter suggests a novel $21^{\text {st }}$ Century take on these old ideas about soul and body. If our selfhood depends constitutively on our beast-machine nature, then instead of the Cartesian motto "cogito ergo sum" one might instead say "I predict (myself), therefore I am".

Closing this chapter, let's return to Andy Clark, and to his as-yet tentative incursions into the territory of consciousness. I wonder how much he really thinks that the "ever-elusive, and ever-familiar, beast that we call 'conscious experience' " (Surfing Uncertainty, p.239) can be naturalized under the PP picture. In particular, does he agree that this 'beast that we call conscious experience' can be made sense of via the beast machine story sketched in this chapter? Or do the conscious aspects of perceptions of the world and of the self remain as elusive as ever, escaping into the night like the beasts of our ancestors' dreams.

\section{Acknowledgements}

For support I am grateful to the Dr. Mortimer and Theresa Sackler Foundation, which supports the Sackler Centre for Consciousness Science, and also to the Canadian Institute for Advanced Research (Azrieli Programme on Mind, Brain, and Consciousness). Many thanks to the Editors of this collection, and to Karl Friston, for very helpful comments on a first draft.

\footnotetext{
${ }^{7}$ It is unclear whether La Mettrie intended to materialise the soul, or to animate or vivify matter. See (Makari, 2016) (especially chapter 11) for an excellent discussion.
} 


\section{References}

Ashby, W. R. (1952). Design for a brain. London, UK: Chapman and Hall.

Ashby, W. R. (1956). An introduction to cybernetics. London, UK: Chapman and Hall.

Barrett, L. F., \& Satpute, A. B. (2017). Neuroscience Letters.

Barrett, L. F., \& Simmons, W. K. (2015). Interoceptive predictions in the brain. Nat Rev Neurosci, 16(7), 419-429. doi:10.1038/nrn3950

Blanke, O., Slater, M., \& Serino, A. (2015). Behavioral, Neural, and Computational Principles of Bodily Self-Consciousness. Neuron, 88(1), 145-166. doi:10.1016/j.neuron.2015.09.029

Botvinick, M., \& Cohen, J. (1998). Rubber hands 'feel' touch that eyes see. Nature, 391(6669), 756. doi:10.1038/35784

Brown, H., Adams, R. A., Parees, I., Edwards, M., \& Friston, K., J. (2013). Active inference, sensory attenuation and illusions. Cogn Process, 14(4), 411-427. doi:10.1007/s10339-013-0571-3

Buckley, C., McGregor, S., Kim, C.-S., \& Seth, A. K. ((in press)). The free-energy principle for perception and action: A mathematical review. Journal of Mathematical Psychology.

Clark, A. (1989). Microcognition: Philosophy, Cognitive Science, and Parallel Distributed Processing. Cambridge, MA: MIT Press.

Clark, A. (1997). Being there. Putting brain, body, and world together again. Cambridge, MA: MIT Press.

Clark, A. (2013). Whatever next? Predictive brains, situated agents, and the future of cognitive science. Behav Brain Sci, 36(3), 181-204. doi:10.1017/S0140525X12000477

Clark, A. (2016). Surfing uncertainty. Oxford: Oxford University Press.

Conant, R., \& Ashby, W. R. (1970). Every good regulator of a system must be a model of that system. International Journal of Systems Science, 1(2), 89-97.

Craig, A. D. (2002). How do you feel? Interoception: the sense of the physiological condition of the body. Nat Rev Neurosci, 3(8), 655-666.

Critchley, H. D., \& Harrison, N. A. (2013). Visceral influences on brain and behavior. Neuron, 77(4), 624-638. doi:10.1016/j.neuron.2013.02.008

Dayan, P., \& Daw, N. D. (2008). Decision theory, reinforcement learning, and the brain. Cogn Affect Behav Neurosci, 8(4), 429-453. doi:10.3758/CABN.8.4.429

de La Mettrie, J. O. (1748). L'homme machine. Leiden: Luzac.

Feldman, H., \& Friston, K. J. (2010). Attention, uncertainty, and free-energy. Front Hum Neurosci, 4, 215. doi:10.3389/fnhum.2010.00215

Felleman, D. J., \& Van Essen, D. C. (1991). Distributed hierarchical processing in the primate cerebral cortex. Cereb Cortex, 1(1), 1-47.

Friston, K. J. (2009). The free-energy principle: a rough guide to the brain? Trends Cogn Sci, 13(7), 293-301.

Friston, K. J. (2010). The free-energy principle: a unified brain theory? Nat Rev Neurosci, 11(2), 127-138. doi:nrn2787 [pii]

$10.1038 / \mathrm{nrn} 2787$

Friston, K. J., Adams, R. A., Perrinet, L., \& Breakspear, M. (2012). Perceptions as hypotheses: saccades as experiments. Frontiers in Psychology, 3, 151. doi:10.3389/fpsyg.2012.00151

Friston, K. J., Daunizeau, J., Kilner, J., \& Kiebel, S. J. (2010). Action and behavior: a free-energy formulation. Biological Cybernetics, 102(3), 227-260. doi:10.1007/s00422-010-0364-z

Friston, K. J., \& Stephan, K. E. (2007). Free-energy and the brain. Synthese, 159(3), 417-458. doi:10.1007/s11229-007-9237-y

Gibson, J. J. (1979). The ecological approach to visual perception. Hillsdale, NJ: Lawrence Erlbaum.

Godfrey-Smith, P. G. (1996). Spencer and Dewey on life and mind. In M. Boden (Ed.), The philosophy of artificial life (pp. 314-331). Oxford: Oxford University Press. 
Hohwy, J. (2013). The Predictive Mind. Oxford: Oxford University Press.

Hutto, D., \& Myin, E. (2013). Radicalizing enactivism: Basic minds without content. Cambridge, MA: MIT Press.

Huys, Q. J., Maia, T. V., \& Frank, M. J. (2016). Computational psychiatry as a bridge from neuroscience to clinical applications. Nat Neurosci, 19(3), 404-413. doi:10.1038/nn.4238

James, W. (1894). The physical basis of emotion. Psychological Review, 1, 516-529.

Makari, G. (2016). Soul machine: The invention of the modern mind. London: W. W. Norton.

Markov, N. T., Ercsey-Ravasz, M., Van Essen, D. C., Knoblauch, K., Toroczkai, Z., \& Kennedy, H. (2013). Cortical high-density counterstream architectures. Science, 342(6158), 1238406. doi:10.1126/science.1238406

Maturana, H., \& Varela, F. (1980). Autopoiesis and Cognition: The Realization

of the Living (Vol. 42). Dordrecht: D. Reidel.

McLeod, P., Reed, N., \& Dienes, Z. (2003). Psychophysics: how fielders arrive in time to catch the ball. Nature, 426(6964), 244-245. doi:10.1038/426244a

Noe, A. (2004). Action in perception. Cambridge, MA: MIT Press.

O'Regan, J. K., \& Noë, A. (2001). A sensorimotor account of vision and visual consciousness. Behav Brain Sci, 24(5), 939-973; discussion 973-1031.

Pezzulo, G. (2014). Why do you fear the bogeyman? An embodied predictive coding model of perceptual inference. Cogn Affect Behav Neurosci, 14(3), 902-911. doi:10.3758/s13415013-0227-x

Pezzulo, G., Rigoli, F., \& Friston, K. (2015). Active Inference, homeostatic regulation and adaptive behavioural control. Prog Neurobiol, 134, 17-35. doi:10.1016/j.pneurobio.2015.09.001

Pinto, Y., van Gaal, S., de Lange, F. P., Lamme, V. A., \& Seth, A. K. (2015). Expectations accelerate entry of visual stimuli into awareness. J Vis, 15(8), 13. doi:10.1167/15.8.13

Powers, A. R., Mathys, C., \& Corlett, P. R. (2017). Pavlovian conditioning-induced hallucinations result from overweighting of perceptual priors. Science, 357(6351), 596-600. doi:10.1126/science.aan3458

Powers, W. T. (1973). Behavior: The control of perception. Hawthorne, NY: Aldine de Gruyter.

Rao, R. P., \& Ballard, D. H. (1999). Predictive coding in the visual cortex: a functional interpretation of some extra-classical receptive-field effects. Nat Neurosci, 2(1), 79-87. doi:10.1038/4580

Rosenfield, L. C. (1968). From beast-machine to man-machine: Animal soul in French letters from Descartes to La Mettrie. New and enlarged edition. New York: Octagon Books.

Schachter, S., \& Singer, J. E. (1962). Cognitive, social, and physiological determinants of emotional state. Psychol Rev, 69, 379-399.

Seth, A. K. (2000). On the Relations Between Behaviour, Mechanism, and Environment: Explorations in Artificial Evolution. (D.Phil.), University of Sussex.

Seth, A. K. (2009). Explanatory correlates of consciousness: Theoretical and computational challenges. Cognitive Computation, 1(1), 50-63.

Seth, A. K. (2013). Interoceptive inference, emotion, and the embodied self. Trends Cogn Sci, 17(11), 565-573. doi:10.1016/j.tics.2013.09.007

Seth, A. K. (2014). A predictive processing theory of sensorimotor contingencies: Explaining the puzzle of perceptual presence and its absence in synesthesia. Cogn Neurosci, 5(2), 97-118. doi:10.1080/17588928.2013.877880

Seth, A. K. (2015a). The cybernetic bayesian brain: from interoceptive inference to sensorimotor contingencies. In J. M. Windt \& T. Metzinger (Eds.), Open MIND (pp. 1-24). Frankfurt A .M: MIND Group.

Seth, A. K. (2015b). Inference to the best prediction. In T. Metzinger \& J. M. Windt (Eds.), Open MIND (pp. 1-24). Frankfurt, a. M.: GER: MIND Group. 
Seth, A. K. ((in press)). From unconscious inference to the Beholder's Share: Predictive perception and human experience. European Review.

Seth, A. K., \& Friston, K. J. (2016). Active interoceptive inference and the emotional brain. Philosophical Transactions of the Royal Society B-Biological Sciences, 371(1708). doi:ARTN 20160007

$10.1098 /$ rstb.2016.0007

Seth, A. K., Suzuki, K., \& Critchley, H. D. (2011). An interoceptive predictive coding model of conscious presence. Frontiers in Psychology, 2, 395. doi:10.3389/fpsyg.2011.00395

Shugg, W. (1968). The cartesian beast-machine in english literature (1663 - 1750). Journal of the History of ideas, 29(2), 279-292.

Sierra, M., \& David, A. S. (2011). Depersonalization: a selective impairment of self-awareness. Consciousness and Cognition, 20(1), 99-108. doi:10.1016/j.concog.2010.10.018

Stephan, K. E., Manjaly, Z. M., Mathys, C. D., Weber, L. A. E., Paliwal, S., Gard, T., . . Petzschner, F. H. (2016). Allostatic Self-efficacy: A Metacognitive Theory of Dyshomeostasis-Induced Fatigue and Depression. Frontiers in human neuroscience, 10(550). doi:10.3389/fnhum.2016.00550

Sterling, P. (2012). Allostasis: a model of predictive regulation. Physiol Behav, 106(1), 5-15. doi:10.1016/j.physbeh.2011.06.004

Suzuki, K., Garfinkel, S. N., Critchley, H. D., \& Seth, A. K. (2013). Multisensory integration across exteroceptive and interoceptive domains modulates self-experience in the rubber-hand illusion. Neuropsychologia, 51(13), 2909-2917. doi:10.1016/j.neuropsychologia.2013.08.014

Thompson, E. (2010). Mind in life: Biology, phenomenology, and the sciences of mind. Cambridge, MA: Harvard University Press.

Tracy, J. L., \& Randles, D. (2011). Four models of basic emotions: A review of Ekman and Cordado, Izard, Levenson, and Panksepp and Watt. Emotion Review, 3(4), 397-405.

Tsakiris, M. (2010). My body in the brain: a neurocognitive model of body-ownership. Neuropsychologia, 48(3), 703-712. doi:S0028-3932(09)00390-X [pii]

10.1016/j.neuropsychologia.2009.09.034

von Helmholtz, H. (1867). Handuch der phsyiologik Optik. Leipzig: Voss.

Wolpert, D. M., \& Kawato, M. (1998). Multiple paired forward and inverse models for motor control. Neural Netw, 11(7-8), 1317-1329.

Young, A. W., \& Leafhead, K. M. (1996). Betwixt life and death: case studies of the Cotard delusion. In P. W. Halligan \& J. C. Marshall (Eds.), Method in Madness. Hove, UK: Psychology Press. 